\title{
The X[[S]]-Sub-Exact Sequence of Generalized Power Series Rings
}

\author{
Wesly Agustinus Pardede ${ }^{1}$, Ahmad Faisol ${ }^{2 *}$ Fitriani $^{3}$ \\ 1,2,3 Universitas Lampung, Indonesia
}

Article Info

Submitted : 28-06-2020

Revised : $01-11-2020$

Accepted : 05-11-2020

Published : 08-11-2020

*Correspondence:

ahmadfaisol@fmipa.unila.ac.id

\section{Abstract}

Let $R$ be a ring, $(S,+, \leq)$ a strictly ordered monoid, and $\mathrm{K}, \mathrm{L}, \mathrm{M}$ are R-modules. Then, we can construct the Generalized Power Series Modules (GPSM) K[[S]], $\mathrm{L}[[\mathrm{S}]]$, and M[[S]], which are the module over the Generalized Power Series Rings (GPSR) R[[S]]. In this paper, we investigate the property of X[[S]]-subexact sequence on GPSM L[[S]] over GPSR R[[S]].

Key Words: , Exact Sequence; Generalized Power Series Module; Generalized Power Series Rings; Strictly Ordered Monoid; X-Sub-Exact Sequence.

http://ejournal.radenintan.ac.id/index.php/al-jabar/index

\section{Introduction}

A non-empty set of $S$ with an associative binary "*" is called a semigroup. If $S$ has an identity element, then $(S, *)$ is called a monoid. Furthermore, if each element of $S$ has an inverse, then $(S, *)$ is called a group (Howie 1995). A ring $(R,+, \cdot)$ is a non-empty set of $\mathrm{R}$ with two binary operations. $(\mathrm{R},+)$ is a commutative group, $(\mathrm{R}, \cdot)$ a semigroup, and satisfies the left and right distributive laws (Adkins and Weintraub 1992).

One example of a ring is the polynomial ring $R[X]$, which is defined as the set of all functions from non-negative integers $\mathbb{N} U\{0\}$ to ring $\mathrm{R}$ with finite support. Furthermore, this ring is generalized into the power series ring $R[X]$ by removing the finite support conditions (Hungerford 1974). Furthermore, the polynomial ring $R[X]$ can be generalized by changing its function domain to any $S$ semigroup. This ring is then known as the semigroup ring and is denoted by $R[S]$ (Gilmer 1984).

A partially ordered relation is a binary relation " $\leq$ " on a non-empty set of $S$ that fulfills reflexive, anti-symmetric, and transitive properties. Furthermore, $(S, \leq)$ is called a partially ordered set. An order " $\leq$ " is said to be trivial if for any $s, t \in S, s \leq t$ results in $s=t$ and is said to be strictly ordered if $(\forall x, y, s \in S)(x<y \rightarrow x+s<y+s)$. Furthermore, $(S, \leq)$ is said to be Artinian if it does not contain any infinite strictly decreasing sequence $s_{1}>s_{2}>s_{3}>\cdots$, and is said to be narrow if it does not contain an infinite subset consisting of pairwise incomparable elements. (Elliott and Ribenboim 1990).

By using the Artinian and narrow partially ordered set concept, ring semigroup R[S] can be generalized into a Generalized Power Series Ring (GPSR) by weakening the finite support condition that became Artinian and narrow. Furthermore, this ring is denoted by $R[[S, \leq]]$ or abbreviated as $R[[S]]$ (Ribenboim 1990). Furthermore, the research results relating to the properties that apply in GPSR can be seen in ((Ribenboim 1991), (Ribenboim 1992), (PriessCrampe and Ribenboim 1993), (Benhissi and Ribenboim 1993), (Ribenboim 1994), (Ribenboim 1995).

Furthermore, the structure of GPSR $R[[S]]$ can be generalized by applying a monoid homomorphism $\omega: S \rightarrow \operatorname{End}(R)$ to the convolution multiplication operation (Mazurek and Ziembowski 2007). This ring is called the Skew Generalized Power Series Ring (SGPSR), and 
it is denoted by $R[[S, \omega]]$. The properties related to the structure of SGPSR $R[[S, \omega]]$ can be seen in ((Mazurek and Ziembowski 2008); (Mazurek and Ziembowski 2009); (Mazurek and Ziembowski 2010), (Faisol 2009), (Faisol 2013), (Faisol 2014), (Faisol, Surodjo, and Wahyuni 2016), (Faisol, Surodjo, and Wahyuni 2018), (Faisol and Fitriani 2019).

It is known that a ring can be seen as a module over itself. Based on this, we can form the Generalized Power Series Module (GPSM) $M[[S]]$, which is a module over GPSR $R[[S]]$ where $M$ is a module over the ring $R$ (Varadarajan 2001a). In addition to the GPSM $M[[S]]$ structure, the necessary and sufficient conditions of $M[[S]]$ to be Noetherian module over $R[[S]]$ can be seen in (Varadarajan 2001b). Furthermore, the generalization of Noetherian property on GPSM $M[[S]]$ can be seen in (Faisol, Surodjo, and Wahyuni 2019a), which is about the necessary and sufficient conditions of GPSM $M[[S]]$ is a $T[[S]]$-Noetherian module. This is obtained by generalizing the necessary and sufficient conditions for the polynomial module $M[[X]]$ to be $S[X]$-Noetherian module over polynomial ring $R[X]$ (Faisol, Surodjo, and Wahyuni 2019c), and applies the relationship between almost generated module, almost Noetherian module and $T$ Noetherian module (Faisol, Surodjo, and Wahyuni 2019b).

The Noetherian properties of an $R$-module $M$ can be investigated through an exact sequence. If there is an exact sequence $A \stackrel{f}{\rightarrow} B \stackrel{g}{\rightarrow} C$ where $A$ and $C$ are Noetherian, then $B$ is a Noetherian $R$-module (Wisbauer 1991). The generalization of the exact sequence in the $R$ module is investigated by (Davvaz and Parnian-Garamaleky 1999). This result is obtained by replacing submodule 0 with submodule $\mathrm{U} \subseteq \mathrm{C}$, called the $\mathrm{U}$-exact sequence. Another study related to the properties of the U-exact sequence can be seen in ((Davvaz and Shabani-Solt 2002) (Anvariyeh and Davvaz 2005)).

Motivated by the U-exact sequence definition, the X-sub-exact sequence concept was introduced in (Fitriani, Surodjo, and Wijayanti 2016), which is a generalization of the exact sequence. Besides that, the generalization of an R-module generator to become a U-generator has been reviewed in (Fitriani, Wijayanti, and Surodjo 2018b). Furthermore, by using the concept of sub-linearly independent modules (Fitriani, Surodjo, and Wijayanti 2017), a basis and free module relative to a family of modules over R can be defined (Fitriani, Wijayanti, and Surodjo 2018a).

It was explained earlier that Varadarajan determines the necessary and sufficient conditions of GPSM M[[S]] is a Noetherian R[[S]]-module; this will be easier to do using the exact sequence concept. Therefore, this motivates us to study the exact sequence of R[[S]]-modules and construct $\mathrm{X}[[\mathrm{S}]]$-sub-exact sequence on GPSM M[[S]]. Besides, this also provides an opportunity to investigate the properties that satisfy them.

\section{The Research Methods}

The research methods are based on the study of literature. They relate to the concept of partially ordered set, strictly ordered monoid, Artinian and narrow properties, generalized power series rings (GPSR), generalized power series modules (GPSM), exact-sequences, and X-subexact sequences. The results of this study obtained by constructing the exact sequence and $\mathrm{X}[[\mathrm{S}]]$-sub-exact sequence over an $\mathrm{R}[[\mathrm{S}]]$-module, as well as investigating the properties that apply in it. 


\section{The Results of the Research and the Discussion}

Before discussing the definition and properties of the $X[[S]]$-sub-exact sequence, the following is explained about the structure of GPSM $M[[S]]$ over GPSR $R[[S]]]$ as well as the exact and $X$-sub-exact sequence definition, which have been explained in ((Ribenboim 1990), (Varadarajan 2001a), (Wisbauer 1991), dan (Fitriani et al. 2016)).

We were given a strictly ordered monoid $(S,+, \leq)$ and commutative ring $R$ with unit element 1. Next, is defined as the set $R[[S]]=\{f: S \rightarrow R \mid \operatorname{supp}(f)$ Artin dan narrow $\}$, with $\operatorname{supp}(f)=\{s \in S \mid f(s) \neq 0\}$. Against the operation of the addition function:

$$
(f+g)(s)=f(s)+g(s)
$$

and convolution multiplication operations:

$$
(f \cdot g)(s)=\sum_{t+u=s} f(t) g(u),
$$

for each $s \in S, t \in \operatorname{supp}(f), u \in \operatorname{supp}(g)$ and $f, g \in R[[S]]$, it can be shown $(R[[S]],+, \cdot)$ is a ring. Furthermore, this ring is called the Generalized Power Series Ring (GPSR).

Furthermore, if given an $R$-module $M$, then the set $M[[S]]=\{\alpha: S \rightarrow$ $M \mid \operatorname{supp}(\alpha)$ Artin dan narrow $\}$ can be formed. Against the operation of the addition function :

$$
(\alpha+\beta)(s)=\alpha(s)+\beta(s)
$$

and scalar multiplication operations:

$$
(\alpha \cdot f)(s)=\sum_{t+u=s} \alpha(t) f(u),
$$

for each $s \in S, t \in \operatorname{supp}(\alpha), u \in \operatorname{supp}(f), f \in R[[\mathrm{~S}]]$, and $\alpha, \beta \in M[[\mathrm{~S}]]$, it can be shown that $M[[S]]$ is an $\mathrm{R}[[\mathrm{S}]]$-module. This module is called the Generalized Power Series Module (GPSM).

The following is the definition of the exact sequence and $X$-sub-exact sequence over an $R$ modules. Let $R$ be a ring and $M_{i}$ an $R$-module for each $i$. $R$-module sequence

$$
\ldots \rightarrow M_{i-1} \stackrel{f_{i}}{\rightarrow} M_{i} \stackrel{f_{i+1}}{\rightarrow} M_{i+1} \rightarrow \cdots
$$

is said to be exact in $M_{i}$ if there are $R$-homomorphism $f_{i}$ and $f_{i+1}$ that satisfies $\operatorname{Im}\left(f_{i}\right)=$ $\operatorname{Ker}\left(f_{i+1}\right)$. The sequence is said to be exact if it is exact at every $M_{i}$.

Furthermore, this exact sequence is generalized to the $X$-sub-exact sequence. Suppose $K$, $L, M$ are modules over $R$ and $X$ is a submodule of $L$. Triple $(K, L, M)$ is said to be $X$-sub-exact over $L$ if there are $R$-homomorphism $f$ and $g$ such that the sequence $K \stackrel{f}{\rightarrow} X \stackrel{g}{\rightarrow} M$ is the exact sequence over $R$-modules.

Next, all submodules $X$ of $L$ can be collected, so the triple $(K, L, M)$ is $X$-sub-exact over $L$. Furthermore, this set is denoted by $\sigma(K, L, M)$. In other words, $\sigma \sigma(K, L, M)=\{X \leq$ $L \mid(K, L, M) X$-sub-exact over $L\}$.

Now, we define the exact sequence of GPSR.

Definition 1. Let $R$ be a ring, $(S, \leq)$ a strictly ordered monoid, and $M_{i}$ modules over $R$ for every $i$. Given GPSR $R[[\mathrm{~S}]]$ and GPSM $M_{i}[[S]]$. An $R[[\mathrm{~S}]]$-module sequence

$$
\ldots \rightarrow M_{i-1}[[\mathrm{~S}]] \stackrel{\mu_{i}}{\rightarrow} M_{i}[[\mathrm{~S}]] \stackrel{\mu_{i+1}}{\rightarrow} M_{i+1}[[\mathrm{~S}]] \rightarrow \cdots
$$

is said to be exact in $M_{i}[[\mathrm{~S}]]$ if there are $R[[S]]$-homomorphisms $\mu_{i}$ and $\mu_{i+1}$ that satisfy $\operatorname{Im}\left(\mu_{i}\right)=\operatorname{Ker}\left(\mu_{i+1}\right)$. Furthermore, this sequence is said to be exact if it is exact at every $M_{i}[[\mathrm{~S}]]$. 
It is known that, if $X$ is the submodule of $M$ over $R$, then the set $X[[S]]=$ $\{\alpha \in M[[S]] \mid \alpha(s) \in X ; \forall s \in S\}$ is the submodule of $M[[S]]$ over $R[[S]]$. The following is the definition of $X[[S]]$-sub-exact sequence of GPSR.

Definition 2. Let $R$ be a ring, $(S, \leq)$ a strictly ordered monoid, and $K, L, M$ are the modules over $R$. Given GPSR $R[[\mathrm{~S}]]$ and GPSM $K[[S]], L[[S]]$ and $M[[S]]$. If $X$ is a submodule of $L$, the triple $(K[[S]], L[[S]], M[[S]])$ is said to be $X[[S]]$-sub-exact over $R[[S]]$ if there are $R[[S]]$ homomorphisms $\mu$ and $\rho$ so that the sequence $K[[S] \stackrel{\stackrel{\mu}{\rightarrow}}{X}[[\mathrm{~S}]] \stackrel{\rho}{\rightarrow} \mathrm{M}[[\mathrm{S}]]$ is the exact sequence over $R[[S]]$.

Based on Definition 2, we can set all $R[[S]]$-submodules $X[[S]]$ of $L[[S]]$ so that triple $(K[[S]], L[[S]], M[[S]])$ is $X[[S]]$-sub-exact over $\mathrm{R}[[\mathrm{S}]]$. This set is then denoted by $\sigma(K[[S]], L[[S]], M[[S]]) \quad$ or $\quad$ written $\quad$ as $\quad \sigma(K[[S]], L[[S]], M[[S]])=\{X[[S]] \leq$ $\mathrm{L}[[\mathrm{S}]] \mid(K[[S]], L[[S]], M[[S]])$ is $X[[\mathrm{~S}]]$-sub-exact over $R[[S]]\}$.

Next, the $X[[S]]$-sub-exact characteristics of GPSR are given as the main results in this study.

Proposition 3. For $i=1,2$, let $K_{i}, L_{i}, M_{i}$ are the modules over $R, X_{i}$ a submodule of $L_{i}$, and $(S, \leq)$ a strictly ordered monoid. If $X_{1}[[\mathrm{~S}]] \in \sigma\left(K_{1}[[S]], L_{1}[[S]], M_{1}[[S]]\right)$ and $X_{2}[[\mathrm{~S}]] \in$ $\sigma\left(K_{2}[[S]], L_{2}[[S]], M_{2}[[S]]\right), \quad$ then $\quad X_{1}[[S]] \times X_{2}[[S]] \in \sigma\left(K_{1}[[S]] \times K_{2}[[S]], L_{1}[[S]] \times\right.$ $\left.L_{2}[[S]], M_{1}[[S]] \times M_{2}[[S]]\right)$.

Proof: Because it is known that $X_{1}[[\mathrm{~S}]] \in \sigma\left(K_{1}[[S]], L_{1}[[S]], M_{1}[[S]]\right)$ and $X_{2}[[\mathrm{~S}]] \in$ $\sigma\left(K_{2}[[S]], L_{2}[[S]], M_{2}[[S]]\right)$, then clearly there is $R[[S]]$-homomorphism $\mu_{1}, \rho_{1}, \mu_{2}$, and $\rho_{2}$ so that $K_{1}[[\mathrm{~S}]] \stackrel{\mu_{1}}{\rightarrow} X_{1}[[S]] \stackrel{\rho_{1}}{\rightarrow} M_{1}[[\mathrm{~S}]]$ and $K_{2}[[\mathrm{~S}]] \stackrel{\mu_{2}}{\rightarrow} X_{2}[[S]] \stackrel{\rho_{2}}{\rightarrow} M_{2}[[\mathrm{~S}]]$ are exact sequences.

Next is defined function $\mu: K_{1}[[S]] \times K_{2}[[S]] \rightarrow X_{1}[[S]] \times X_{2}[[S]]$, where $\mu\left(\left(\alpha_{1}, \alpha_{2}\right)\right)=$ $\left(\mu_{1}\left(\alpha_{1}\right), \mu_{2}\left(\alpha_{2}\right)\right)$, for each $\left(\alpha_{1}, \alpha_{2}\right) \in K_{1}[[\mathrm{~S}]] \times K_{2}[[\mathrm{~S}]]$ and $\rho: X_{1}[[\mathrm{~S}]] \times X_{2}[[\mathrm{~S}]] \rightarrow M_{1}[[S]] \times$ $M_{2}[[S]]$, where $\rho\left(\left(\beta_{1}, \beta_{2}\right)\right)=\left(\rho_{1}\left(\beta_{1}\right), \rho_{2}\left(\beta_{2}\right)\right)$, for each $\left(\beta_{1}, \beta_{2}\right) \in X_{1}[[S]] \times X_{2}[[S]]$.

Based on the definitions of the functions $\mu$ and $\rho$, it can be shown easily that the functions $\mu$ and $\rho$ are $R[[S]]$-homomorphisms. Therefore, the sequence $K_{1}[[\mathrm{~S}]] \times K_{2}[[\mathrm{~S}]] \stackrel{\mu}{\rightarrow} X_{1}[[S]] \times$ $X_{2}\left[[S] \stackrel{\rho}{\rightarrow} M_{1}[[S]] \times M_{2}[[S]]\right.$ is an exact sequence. In other words, $X_{1}[[\mathrm{~S}]] \times X_{2}[[\mathrm{~S}]] \in$ $\sigma\left(K_{1}[[S]] \times K_{2}[[S]], L_{1}[[S]] \times L_{2}[[S]], M_{1}[[S]] \times M_{2}[[S]]\right)$.

As a direct result of Proposition 3, the following properties are obtained for a set of indexes $\Delta$.

Corollary 4. Let $K_{\delta}[[S]], L_{\delta}[[S]], M_{\delta}[[S]]$ are a family of $R[[\mathrm{~S}]]$-module and $X_{\delta}[[\mathrm{S}]]$ is a submodule of $L_{\delta}[[S]]$ for every $\delta \in \Delta$. If $X_{\delta}[[S]] \in \sigma\left(K_{\delta}[[S]], L_{\delta}[[S]], M_{\delta}[[S]]\right)$ for every $\delta \in$ $\Delta$, then $\prod_{\delta \in \Delta} X_{\delta}[[\mathrm{S}]] \in \sigma\left(\prod_{\delta \in \Delta} K_{\delta}[[S]], \prod_{\delta \in \Delta} L_{\delta}[[S]], \prod_{\delta \in \Delta} M_{\delta}[[S]]\right)$

The following properties show that if triple $(0, L[[S]], M[[S]]) X_{1}[[S]]$-sub-exact and dan also $X_{2}[[\mathrm{~S}]]$-sub-exact, then triple $(0, L[[S]], M[[S]])$ is $\left(X_{1}[[\mathrm{~S}]] \cap X_{2}[[\mathrm{~S}]]\right)$-sub-exact over $R[[S]]$. 
Proposition 5. Suppose that $L$ and $M$ are modules over $\mathrm{R}$ and $(\mathrm{S}, \leq)$ a strictly ordered monoid. Given $R[[S]]$-modules $L[[S]]$ and $M[[S]]$, and $X_{1}[[\mathrm{~S}]], X_{2}[[\mathrm{~S}]]$ are submodules of $L[[S]]$. If $X_{1}[[\mathrm{~S}]], X_{2}[[\mathrm{~S}]] \in \sigma(0, L[[S]], M[[S]])$, then $X_{1}[[\mathrm{~S}]] \cap X_{2}[[\mathrm{~S}]] \in \sigma(0, L[[S]], M[[S]])$.

Proof: Since $X_{1}[[\mathrm{~S}]], X_{2}[[\mathrm{~S}]] \in \sigma(0, L[[S]], M[[S]])$, then there are $R[[S]]$-homomorphisms $\rho_{1}$ and $\rho_{2}$ such that $0 \rightarrow X_{1}[[\mathrm{~S}]] \stackrel{\rho_{1}}{\rightarrow} M[[S]]$ and $0 \rightarrow X_{2}[[\mathrm{~S}]] \stackrel{\rho_{2}}{\rightarrow} M[[S]]$ are exact sequences. Therefore, $\rho_{1}$ and $\rho_{2}$ are $R[[S]]$-monomorphisms. Next, it is defined as $\rho=\rho_{1} \mid X_{1}[[\mathrm{~S}]] \cap X_{2}[[\mathrm{~S}]]$. Then, $\rho$ is an $R[[S]]$-monomorphism. Therefore, $0 \rightarrow X_{1}[[\mathrm{~S}]] \cap X_{2}[[\mathrm{~S}]] \stackrel{\rho_{1}}{\rightarrow} M[[S]]$ is an exact sequence. So, it is proved that $X_{1}[[\mathrm{~S}]] \cap X_{2}[[\mathrm{~S}]] \in \sigma(0, L[[S]], M[[S]])$.

The properties described in Proposition 5 cause the following properties to take the consequence.

Corollary 6. Suppose $L$ and $M$ are modules over $R$, and $(S, \leq)$ is strictly ordered monoid. Given GPSM $L[[\mathrm{~S}]]$ and $M[[\mathrm{~S}]]$ over GPSR $R[[S]]$, and $X_{\delta}[[\mathrm{S}]]$ is a submodule of $M[[S]]$ for every $\delta \in$ $\Delta$. If $X_{\delta}[[\mathrm{S}]] \in \sigma(0, L[[\mathrm{~S}]], M[[\mathrm{~S}]])$ for each $\delta \in \Delta$, then $\bigcap_{\delta \in \Delta} X_{\delta}[[\mathrm{S}]] \in \sigma(0, L[[\mathrm{~S}]], M[[\mathrm{~S}]])$

Example 7. After the properties related to $X[[S]]$-sub-exact sequence of GPSR are given, here are examples:

1. Triple $(R[X], R[X], 0)$ is $R$-sub-exact on $R[X]$, where $R$-homomorphism $f: R[X] \rightarrow R$ is defined by

$$
f\left(a_{0}+a_{1} x+\cdots+a_{n} x^{n}\right)=a_{0}
$$

and $g$ is zero mappings, such that $R[X] \stackrel{f}{\rightarrow} R \stackrel{g}{\rightarrow} 0$ is an exact sequence.

2. Triple $(R[X], R[X], R[X])$ is a 0 -sub-exact on $R[X]$, because $R[X] \stackrel{g}{\rightarrow} 0 \stackrel{i}{\rightarrow} R[X]$ is an exact sequence, where the zero mapping $g$ and inclusion $i$ are $R$-homomorphisms.

3. If $I[[S]]$ is ideal of $R[[S]]$, then we can form the exact sequence $I[[S]] \stackrel{i}{\rightarrow} R[[S]] \stackrel{\pi}{\rightarrow} R[[S]] / I[[S]]$, where $i$ is an identity and dan $\pi$ a natural homomorphism.

\section{Conclusion and Suggestion}

If given GPSM $K[[S]], L[[S]], M[[S]]$ over GPSR $R[[S]]$, then we can form a set of all submodule $X[[S]]$ of $L[[S]]$ so that triple $(K[[S]], L[[S]], M[[S]])$ is $X[[S]]$-sub-exact.

If $\quad X_{\delta}[[\mathrm{S}]] \in \sigma\left(K_{\delta}[[S]], L_{\delta}[[S]], M_{\delta}[[S]]\right) \quad$ for $\quad$ each $\quad \delta \in \Delta, \quad$ then $\quad \prod_{\delta \in \Delta} X_{\delta}[[S]] \in$ $\sigma\left(\prod_{\delta \in \Delta} K_{\delta}[[\mathrm{S}]], \prod_{\delta \in \Delta} L_{\delta}[[\mathrm{S}]], \prod_{\delta \in \Delta} M_{\delta}[[S]]\right)$. If $X_{\delta}[[\mathrm{S}]] \in \sigma(0, L[[\mathrm{~S}]], M[[\mathrm{~S}]])$ for each $\delta \in \Delta$, then $\bigcap_{\delta \in \Delta} X_{\delta}[[\mathrm{S}]] \in \sigma(0, L[[\mathrm{~S}]], M[[\mathrm{~S}]])$.

In this paper, there are still many opportunities to investigate the characterization of the $X[[S]]$-sub-exact sequence of GPSR $R[[S]]$. Also, investigating the necessary and sufficient conditions for $X[[S]]$ to be a Noetherian module over $R[[S]]$, where $K[[S]], M[[S]]$ are Noetherian modules, but $L[[S]]$ is not Noetherian. 


\section{References}

Adkins, William A., and Steven H. Weintraub. 1992. Algebra: An Approach via Module Theory. New York: Springer-Verlag, New York, Inc.

Anvariyeh, S. M., and B. Davvaz. 2005. “On Quasi-Exact Sequences.” Bull. Korean Math. Soc. 42:149-55.

Benhissi, A., and Paulo Ribenboim. 1993. "Ordered Rings of Generalized Power Series.” in Ordered Algebraic Structures.

Davvaz, B., and Y. A. Parnian-Garamaleky. 1999. “A Note on Exact Sequences.” Bulletin of The Malaysian Mathematical Society 22:53-56.

Davvaz, B., and H. Shabani-Solt. 2002. “A Generalization of Homological Algebra.” Journal of the Korean Mathematical Society 39(6):881-98.

Elliott, G. A., and Paulo Ribenboim. 1990. "Fields of Generalized Power Series." Archiv Der Mathematik 54(4):365-71.

Faisol, Ahmad. 2009. "Homomorfisam Ring Deret Pangkat Teritlak Miring.” Jurnal Sains MIPA 15(2):119-24.

Faisol, Ahmad. 2013. "Pembentukan Ring Faktor Pada Ring Deret Pangkat Teritlak Miring.” Pp. 1-5 in Prosiding Semirata FMIPA Univerisitas Lampung.

Faisol, Ahmad. 2014. "Endomorfisma Rigid Dan Compatible Pada Ring Deret Pangkat Tergeneralisasi Miring." Jurnal Matematika 17(2):45-49.

Faisol, Ahmad, and Fitriani. 2019. "The Sufficient Conditions for Skew Generalized Power Series Module M[[S,w]] to Be T[[S,w]]-Noetherian R[[S,w]]-Module." Al-Jabar: Jurnal Pendidikan Matematika 10(2):285-92.

Faisol, Ahmad, Budi Surodjo, and Sri Wahyuni. 2016. "Modul Deret Pangkat Tergeneralisasi Skew T-Noether." Pp. 95-100 in Prosiding Seminar Nasional Aljabar, Penerapan dan Pembelajarannya. Sanata Dharma University Press.

Faisol, Ahmad, Budi Surodjo, and Sri Wahyuni. 2018. "The Impact of the Monoid Homomorphism on The Structure of Skew Generalized Power Series Rings.” Far East Journal of Mathematical Sciences (FJMS) 103(7):1215-27.

Faisol, Ahmad, Budi Surodjo, and Sri Wahyuni. 2019a. "T[[S]]-Noetherian Property on Generalized Power Series Modules." JP Journal of Algebra, Number Theory and Applications 43(1):1-12.

Faisol, Ahmad, Budi Surodjo, and Sri Wahyuni. 2019b. "The Relation between Almost Noetherian Module, Almost Finitely Generated Module and T-Noetherian Module." in Journal of Physics: Conference Series. Vol. 1306. Institute of Physics Publishing.

Faisol, Ahmad, Budi Surodjo, and Sri Wahyuni. 2019c. "The Sufficient Conditions for R[X]Module M[X] to Be S[X]-Noetherian.” 5(1):1-13.

Fitriani, Budi Surodjo, and Indah Emilia Wijayanti. 2016. “On Sub-Exact Sequences.” Far East Journal of Mathematical Sciences 100(7):1055-65.

Fitriani, Budi Surodjo, and Indah Emilia Wijayanti. 2017. “On X-Sub-Linearly Independent Modules.” Journal of Physics: Conference Series 893(1). 
Fitriani, Indah Emilia Wijayanti, and Budi Surodjo. 2018a. "A Generalization of Basis and Free Modules Relatives to a Family of R-Modules." Journal of Physics: Conference Series 1097(1).

Fitriani, Indah Emilia Wijayanti, and Budi Surodjo. 2018b. "Generalization of U-Generator and M-Subgenerator Related to Category $\sigma[\mathrm{M}]$. . Journal of Mathematics Research 10(4):1016.

Gilmer, Robert. 1984. Commutative Semigroup Rings. Chicago and London: The University of Chicago.

Howie, John M. 1995. Fundamentals of Semigroup Theory. New York: Oxford University Press Inc.

Hungerford, Thomas W. 1974. Algebra. New York: Springer-Verlag New York, Inc.

Mazurek, Ryszard, and Michal Ziembowski. 2010. "Weak Dimension and Right Distributivity of Skew Generalized Power Series Rings." Journal of the Mathematical Society of Japan 62(4):1093-1112.

Mazurek, Ryszard, and Michał Ziembowski. 2007. "Uniserial Rings of Skew Generalized Power Series.” Journal of Algebra 318(2):737-64.

Mazurek, Ryszard, and Michał Ziembowski. 2008. "On von Neumann Regular Rings of Skew Generalized Power Series.” Communications in Algebra 36(5):1855-68.

Mazurek, Ryszard, and Michał Ziembowski. 2009. "The Ascending Chain Condition for Principal Left or Right Ideals of Skew Generalized Power Series Rings." Journal of Algebra 322(4):983-94.

Priess-Crampe, S., and Paulo Ribenboim. 1993. "Fixed Points, Combs and Generalized Power Series." Abhandlungen Aus Dem Mathematischen Seminar Der Universität Hamburg 63:227-44.

Ribenboim, Paulo. 1990. "Generalized Power Series Rings." in Lattices, Semigroups, and Universal Algebra.

Ribenboim, Paulo. 1991. "Rings of Generalized Power Series: Nilpotent Elements." Abh. Math. Sem. Univ. Hamburg 61:15-33.

Ribenboim, Paulo. 1992. "Noetherian Rings of Generalized Power Series." Journal of Pure and Applied Algebra 79(3):293-312.

Ribenboim, Paulo. 1994. "Rings of Generalized Power Series II: Units and Zero-Divisors." Journal of Algebra 168:71-89.

Ribenboim, Paulo. 1995. "Special Properties of Generalized Power Series." Journal of Algebra 173:566-86.

Varadarajan, K. 2001a. "Generalized Power Series Modules." Communications in Algebra 29(3):1281-94.

Varadarajan, K. 2001b. "Noetherian Generalized Power Series Rings and Modules." Communications in Algebra 29(1):245-51. 
Wisbauer, Robert. 1991. Foundations of Module and Ring Theory. D"usseldorf: Gordon and Breach Science Publishers. 